\title{
Transport in finite size systems: an exit time approach
}

\author{
P. Castiglione ${ }^{(a)(b)}$, M. Cencini ${ }^{(a)}$, A. Vulpiani $^{(a)}$ and E. Zambianchi ${ }^{(c)}$ \\ (a) Dipartimento di Fisica, Università "La Sapienza", and INFM, Unità di Roma 1, \\ P.le A. Moro 2, I-00185, Roma, Italy \\ (b) Present address: Laboratoire de Physique Statistique, Ecole Normale Supérieure, \\ 24 rue Lhomond, 75231 Paris, France \\ (c) Istituto di Meteorologia e Oceanografia, Istituto Universitario Navale, \\ Via Acton 38, I-80133 Napoli, Italy
}

(September 3, 2018)

\begin{abstract}
In the framework of chaotic scattering we analyze passive tracer transport in finite systems. In particular, we study models with open streamlines and a finite number of recirculation zones. In the non trivial case with a small number of recirculation zones a description by mean of asymptotic quantities (such as the eddy diffusivity) is not appropriate. The non asymptotic properties of dispersion are characterized by means of the exit time statistics, which shows strong sensitivity on initial conditions. This yields a probability distribution function with long tails, making impossible a characterization in terms of a unique typical exit time.
\end{abstract}

\section{INTRODUCTION}

The problem of transport in velocity fields characterized by different flow regimes in different subareas (i.e. a "spatially disordered set of streamlines" [四]) has been considered by many authors. Particular attention has been devoted to steady and time-dependent oceanic and atmospheric flows with recirculations [2 25] and the related problem of the dispersion in porous media [6 8]. In this paper we will focus our attention on flows with recirculations of geophysical interest. The study of transport properties in presence of recirculations has a crucial relevance, since gyre- or eddy-like recirculating patterns are ubiquitous features in different areas of the world ocean and atmosphere. In the ocean these features are typically induced by forcing spatial structures at the boundaries (e.g. bottom topography, wind stress curl, coast-lines) or by intrinsic dynamical reasons (mesoscale eddies), one interacting with the other (for a general reference see [9]).

It is worth noting that large-scale meandering jets, which are typically associated with the extensions of western boundary currents, often separate ocean regions characterized by different physical and biogeochemical characteristics. Consequently, the study of mixing processes in correspondence of them is important also for multidisciplinary investigations [10] 
(e.g. the biological effects of longitudinal transport in western boundary current extensions see [11]).

The systems we consider are characterized by the joint presence of open streamline areas where particle motion is essentially a ballistic flight and closed streamline regions typically distributed according to a periodic geometry, where particles tend to be trapped. The easiest way to study the transport properties in such systems is by averaging over smaller space or over shorter time scales. Typically, this results in the possibility of describing dispersion by an equation for macroscopic quantities of the system such as the average passive scalar concentration in terms of an effective drift and an effective diffusivity (as classically done by Taylor [12]; see [1], for a detailed discussion), at least in the case of standard diffusion. However, a description in terms of equations for macroscopic quantities needs to average out the small scale (fast) features of the velocity field and thus such approach applies only for asymptotic times, when particles have been able to thoroughly sample the different flow regimes in the system.

As stressed by Young [1], before reaching this asymptotic regime very interesting transient behaviors, which cannot be described within an effective diffusion model, could occur. The transient regime may be very long and, if the system is finite, the asymptotic one may not be reached. This is, typically, the case of finite domain systems with no large scale separation between the size of the domain and the largest characteristic Eulerian length. In realistic flows, which are usually characterized by a fairly limited number of recirculations, fluid particles ordinarily sample just a fraction of the available regimes.

Therefore, it is often not possible to characterize dispersion simply in terms of asymptotic quantities such as average velocity and diffusion coefficients: different approaches are needed, as done in [13 15] for the characterization of transport in closed domains; such as the symbolic dynamics approach to the sub-diffusive behavior in a stochastic layer and to mixing in meandering jets respectively described in [16] and [17]; or the study of tracer dynamics in open flows in terms of chaotic scattering by Tél and coworkers [18 20]; and the exit time approach of [5].

The aim of this paper is to describe dispersion in finite size systems. In particular, we want to characterize flows with a small number of recirculations using some ideas stemming from the chaotic scattering theory.

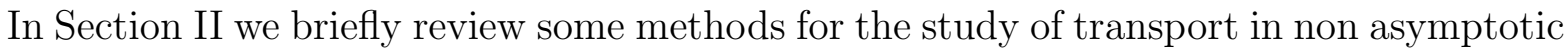
regimes, namely the finite-size diffusion coefficients and the exit time statistics originated from the chaotic scattering phenomenon. Section [II] contains a description of the two studied models (traveling wave and meandering jet) and some numerical results. Section $\mathbb{I V}$ is devoted to the comparison between numerical results and a probabilistic model. Conclusions are presented in Section $\square$.

\section{TOOLS FOR THE STUDY OF NON-ASYMPTOTIC TRANSPORT PROPERTIES}

The investigation of passive tracer diffusion is usually reduced to the study of an effective equation describing the long-time, large-distance average tracer concentration behavior. Under rather general hypotheses, given an Eulerian velocity field the long-time transport process is uniquely characterized by the effective diffusion (or diffusivity) tensor $D^{E}{ }_{i j}$ : 


$$
D^{E}{ }_{i j}=\lim _{t \rightarrow \infty} \frac{1}{2 t}<\left(x_{i}(t)-<x_{i}>\right)\left(x_{j}(t)-<x_{j}>\right)>,
$$

where $\mathbf{x}(t)$ is the position of the tracer particle at time $t ; i, j=1, \cdots, d$, and $d$ is the spatial dimension; the average is taken over the tracer initial conditions or, equivalently, over an ensemble of tracer particles. The effective diffusion tensor $D_{i j}^{E}$ takes into account the molecular diffusivity and the details of the velocity field. Even in presence of simple Eulerian fields (e.g. laminar and periodic in time) the diffusion coefficient as a function of the parameters of the velocity field can display a rather non trivial behavior [21,22]

It is worth underlining that the diffusivity tensor (11) is mathematically well defined only in the asymptotic limit, therefore its use in finite size domains yields meaningful results only if the characteristic length $l_{u}$ of the velocity field is much smaller than the size of the domain. If this is not the case (e.g. in many geophysical settings or plasma physics [13]), dispersion can be characterized more satisfactorily using concepts and techniques borrowed by the dynamical systems theory which will be discussed in the following.

As an example, consider the relative diffusion of a cloud of $\mathcal{N}$ test particles in a smooth velocity field with characteristic length $l_{u}$ and assume that the Lagrangian motion is chaotic. Denoting with $R(t)$ the radius of the cloud:

$$
R^{2}(t)=\frac{1}{\mathcal{N}} \sum_{k=1}^{\mathcal{N}}\left|\mathbf{x}_{k}(t)-\langle\mathbf{x}(t)\rangle\right|^{2}
$$

with

$$
\langle\mathbf{x}(t)\rangle=\frac{1}{\mathcal{N}} \sum_{n=1}^{\mathcal{N}} \mathbf{x}_{n}(t)
$$

we expect the following regimes to hold

$$
\overline{R^{2}(t)} \simeq \begin{cases}R^{2}(0) \exp (L(2) t) & \text { if }{\overline{R^{2}(t)}}^{1 / 2} \ll l_{u} \\ 2 D^{E} t & \text { if } \bar{R}^{2}(t)\end{cases}
$$

where $L(2) \geq 2 \lambda$ is the generalized Lyapunov exponent [23], $D^{E}$ is the diffusion coefficient under the assumption of isotropy, $D^{E}{ }_{i j}=\delta_{i j} D^{E}$, and the over-bar denotes the average over initial conditions of the cloud. Equation (4) states that as long as the size of the cloud is relatively small, chaotic behavior prevails; later on, dispersion displays a diffusive behavior.

Another way to describe the above behavior, even though at first glance rather artificial, is by introducing the "doubling time" $\mathcal{T}(\delta)$ at scale $\delta$ as follows: define a series of thresholds $\delta^{(n)}=r^{n} \delta^{(0)}$, where $\delta^{(0)}$ is the initial size of the cloud, and then measure the time $T\left(\delta^{(0)}\right)$ it takes for the growth from $\delta^{(0)}$ to $\delta^{(1)}=r \delta^{(0)}$, and so on for $T\left(\delta^{(1)}\right), T\left(\delta^{(2)}\right), \ldots$ up to the largest considered scale. Though strictly speaking the term "doubling time" refers to the threshold rate $r=2$, any value can be chosen for $r$, even if a too large one might not separate different scale contributions.

Performing $\mathcal{M} \gg 1$ experiments with different initial conditions for the cloud, we define an average doubling time $\mathcal{T}(\delta)$ at scale $\delta$ as

$$
\mathcal{T}(\delta)=<T(\delta)>_{\mathcal{M}}=\frac{1}{\mathcal{M}} \sum_{m=1}^{\mathcal{M}} T_{m}(\delta) .
$$


It is worth noting that the average in (5) is different from the usual time average (see [24] for a detailed discussion of this point). A finite size diffusion coefficient $D(\delta)$ can then be introduced as

$$
D(\delta)=\delta^{2} \lambda(\delta)
$$

where

$$
\lambda(\delta)=\frac{\ln r}{\mathcal{T}(\delta)}
$$

is the finite size Lagrangian Lyapunov exponent [14]. It can be shown that the Lyapunov exponent $\lambda$ can be obtained from $\lambda(\delta)$ for $\delta \rightarrow 0$, namely $\lambda(\delta)=\lambda$ for $\delta \ll l_{u}$.

For a tracer cloud of non-infinitesimal size $\mathcal{T}(\delta)$ depends on the details of the nonlinear mechanisms of expansion: in the case of standard diffusion $D(\delta)$ is a constant, i.e. $1 / \mathcal{T}(\delta) \sim$ $\delta^{-2}[14$. Thus

$$
\lambda(\delta) \simeq \begin{cases}\lambda & \text { if } \delta \ll l_{u} \\ D / \delta^{2} & \text { if } \delta \gg l_{u}\end{cases}
$$

The fixed scale analysis allows us to extract physical information at different spatial scales avoiding some unpleasant consequences resulting from working at a fixed delay time $t$. For instance, in presence of strong intermittency, $R^{2}(t)$ as a function of $t$ can be rather different from one realization to another. In figure 1a we present an example taken from 114 where different exponential rates of growth for different realizations of $R^{2}(t)$ produce a spurious behavior of $\overline{R^{2}(t)}$. Since at small scales, say $l_{u}$, one typically has an exponential growth of $R(t)$ due to Lagrangian chaos and at large scale a diffusive behavior, one can mimic $R(t)$ as follows:

$$
R^{2}(t)= \begin{cases}\delta_{0}^{2} e^{2 \gamma t} & R(t)<l_{u} \\ 2 D\left(t-t_{*}\right) & R(t)>l_{u}\end{cases}
$$

where $t_{*}$ is such that $R(t)$ is continuous.

Figure 1b shows the average $\overline{R^{2}(t)}$ versus time $t$; at large times the diffusive behavior is recovered, but at intermediate times an apparently anomalous regime occurs. This last regime is only due to the superposition of exponential and diffusive contributions by different samples at the same time. On the other hand the doubling time analysis yields unambiguous results, see figure $1 \mathrm{c}$.

Let us remark that the above technique recovers the usual asymptotic description when there is a large scales separation and also if a genuine anomalous diffusion occurs [25], in addition, it constitutes a systematic method to treat situations in which the scales are not well separated. Moreover, the finite size diffusion coefficient $D(\delta)$ enables the understanding of the different spreading mechanisms at different scales. This has been recently shown in [15], where this method is applied to analyzing experimental trajectories described by surface drifters in the Adriatic Sea.

Another interesting approach to the study of transport properties in finite systems is the chaotic scattering theory used in [18 20] for passive tracer advection in open flows. In a nutshell, chaotic scattering can be summarized as follows. A particle arriving from, say, 
$x=-\infty$ enters a region (defined as the scattering, or interacting, region) where due to the presence of a potential it scatters, then exits and goes to $x=\infty$. Typically, for a rather general class of potentials [26] the time a particle takes to escape from the scattering region can be very sensitive on the impact parameter $b$ and thus displaying a chaotic character (like a ball in a pinball game). This justifies the definition in terms of chaos, even if since chaos is a time-asymptotic concept, from a technical point of view this kind of behavior is not chaotic: particles after a transient (even if very long) leave the scattering region and enter a regular motion regime.

A nice example of the application of chaotic scattering theory in passive tracer study is given in [18,19] for the motion of Lagrangian tracers in blinking vortex-sink system and in a von Karman vortex street behind a cylinder in a channel. Tracer particles can temporarily be trapped in certain regions, e.g. the wakes of the von Karman street, performing very irregular paths. On the other hand, since the non stationarity of the flow is mainly restricted to a finite mixing region around the obstacles, the asymptotic almost free particle motion is also recovered.

For the use of the exit time approach for the transport and mixing in volume preserving maps see Ref. [27].

The analogy between chaotic scattering, occurring in Hamiltonian systems, and passive scalar motion can be drawn in formal terms for two dimensional incompressible velocity field [18. In this case the Eulerian field is described by a stream-function $\psi(x, y, t)$, and the corresponding equations for the Lagrangian evolution are:

$$
\frac{\mathrm{d} x}{\mathrm{~d} t}=-\frac{\partial \psi(x, y, t)}{\partial y}, \frac{\mathrm{d} y}{\mathrm{~d} t}=\frac{\partial \psi(x, y, t)}{\partial x} .
$$

The equations (10) are nothing but the canonical equations for a one dimensional time dependent Hamiltonian system, where the stream function plays the role of the Hamiltonian.

Since in chaotic scattering the particle exit time from the interacting region depends strongly on the initial conditions, it is interesting to look at the time delay function [18], i.e. the exit time as a function of the initial position, $\tau(x(0), y(0))$, e.g. with $x(0)=x_{0}$. In presence of chaotic scattering $\tau\left(x_{0}, y(0)\right)$, displays a rather irregular shape (see, e.g., fig. 5.18 in [26]).

As already remarked, since in chaotic scattering the irregular character is confined both in space and time (i.e. one has the so-called transient chaos), the Lyapunov exponent is trivially zero; however, a sort of high sensitivity on initial conditions is suggested by the occurrence of very different time delays for very close deployment locations.

The presence of large excursions for the exit time has an obvious relevance for transport processes in finite size systems. The wild variations of $\tau\left(x_{0}, y(0)\right)$ pose severe limits on the possibility to make prediction on the particles behavior and force us to use statistical approaches. This leads to introduce the probability distribution function, $P(\tau)$, of the exit times $\tau\left(x_{0}, y(0)\right)$.

\section{TWO SIMPLE FLOWS}

In this paper two idealized flow models are studied; both are reminiscent of oceanographic features. We consider finite amplitude Rossby waves in a channel, recently revisited in 
[28] (see also [29,30]). In the reference frame moving with the phase speed of the wave, the flow field shows a central open streamline region (ballistic motion) sided by trapping recirculations (see fig. 2a); this flow pattern is a suitable, even if simplified, prototype for studying the effect of a finite number of trapping areas on the longitudinal dispersion of particles.

The two-dimensional incompressible Rossby wave flow [28] here considered is specified by the following stream-function:

$$
\Psi_{0}(x, y)=A_{0} \sin \left(K_{0} x\right) \sin \left(L_{0} y\right)-c_{0} y
$$

where $A_{0}$ is related to the maximum velocity in the $y$-direction, $\left(K_{0}, L_{0}\right)$ is the wave vector and $c_{0}$ is the phase speed of the primary wave in the $x$-direction. In (11) $\Psi_{0}$ is expressed in the reference frame co-moving with the primary wave.

In order to reproduce the instabilities usually present in geophysical flows, we introduce a time-periodic perturbation, $\delta \Psi(x, y, t)$ :

$$
\delta \Psi(x, y, t)=\alpha \sin \left(K_{1} x-\Omega t\right) \sin \left(L_{1} y\right)
$$

where $\alpha>0$ is a (not necessarily small) parameter which controls the amplitude of the perturbation and $\left(K_{1}, L_{1}\right)$ is the wave-vector of the perturbation (secondary wave). Even though realistic disturbances cannot be characterized in terms of a single wave alone [31], the perturbation (12) is a first step towards a description of the complex structure of transport mechanisms in these systems.

The second flow we investigate is a meandering jet, which represents a natural extension of the above Rossby wave system, and it was extensively studied in the literature with particular reference to the Gulf Stream [10,32. Again, the flow can be subdivided into different regions roughly corresponding to a prograde flow (in reality, the current jet core; in our schematization, the open streamline regime), recirculation regions and, at a farther distance, an essentially quiescent (far) field (see fig. 2b).

We consider now fluid particle trajectories in the two-dimensional kinematic model originally proposed by Bower [33] and thereafter widely studied [17,34]36]. The large-scale flow, in a reference frame moving eastward with a velocity coinciding with the meander phase speed and suitably nondimensionalized, is expressed by the stream function:

$$
\psi(x, y)=-\tanh \left[\frac{y-B \cos k x}{\left(1+k^{2} B^{2} \sin ^{2} k x\right)^{1 / 2}}\right]+c y .
$$

In fig. $2 \mathrm{~b}$ we show the streamlines in the co-moving frame.

As mentioned above, chaotic advection may be introduced trough a time dependence. Among the different mechanisms discussed in [34], we chose here a time-periodic oscillation of the meander amplitude:

$$
B(t)=B_{0}+\gamma \cdot \cos (\omega t+\theta),
$$

where we set $B_{0}=1.2, \gamma=0.3, \omega=0.4$ and $\theta=\pi / 2$. The parameters choice is mainly motivated by observations and numerical results, as discussed in [17].

It is worth underlining that, even if quite a great deal of efforts has been devoted to study fluid exchange across the jet [33 35], very little is known as to tracer behavior in the 
along-jet direction (periodic flows with open streamlines have been proposed as model for the meandering jets; the presence of recirculations has been seen to induce non-trivial effects on the along-jet dispersion, see [37).

Tracer particle trajectories have been numerically generated from eqs. (10) with the stream-functions corresponding to the traveling wave (111-12) and the meandering jet (1514). However, since the results relative to the two flows are qualitatively the same, we shall present and discuss just those obtained for the meandering jet.

As can be seen from the streamfunctions, the two flows are periodic in the longitudinal direction. Since we are interested in the characterization of longitudinal transport, the number of elementary flow structures (or cells), $N_{c}$, constituting the system, is a crucial parameter. For very large $N_{c}$, the dispersion properties of the system can be studied using asymptotic techniques, e.g. the multiscale method [38,39]. On the contrary, we mainly concentrate on systems with a small number of cells, namely $N_{c} \simeq 2-10$.

The first focus of our analysis is the particle exit time (or time delay function, see Sect. 【II) as a function of the initial position, i.e. the time $\tau\left(x_{0}, y(0)\right)$ a tracer particle deployed at $\left(x_{0}, y(0)\right)$ takes to reach the boundary $x_{\max }=N_{c} 2 \pi / k$. Two very different scenarios occur for large and small $N_{c}$.

Figs. 3a-d show the behavior of $\tau\left(x_{0}, y(0)\right)$ for $N_{c}=3,10,100,1000$. Increasing the system size there is a clear change in the shape of $\tau\left(x_{0}, y(0)\right)$ : highly inhomogeneous structures (fractal objects) for low $N_{c}$ (figs. 3a,b), with very strong fluctuations of the exit time value even for small variations of the initial conditions. As $N_{c}$ increases, the shape of $\tau\left(x_{0}, y(0)\right)$ becomes more and more homogeneous.

The fractal character of $\tau\left(x_{0}, y(0)\right)$ is evident from figs. 3a and the enlargements figs. 4a-b, which suggest the self-similarity of the structures at different scales. This can be quantitatively assessed studying the correlation dimension $\mathcal{D}$ of the initial condition set $\{y(0)\}$ such that $\tau\left(x_{0}, y(0)\right)>\Theta$. Using the Grassberger and Procaccia algorithm 40, i.e. computing the percentage $C(r)$ of pairs $\left(y_{i}, y_{j}\right)$ such that $\left|y_{i}-y_{j}\right| \leq r$; for small $r$ we obtain $C(r) \sim r^{\mathcal{D}}$ (shown in fig. 5) with $\mathcal{D}<1$. The value of $\mathcal{D}$ can depend weakly on the threshold $\Theta$, e.g. for $\Theta=15$ and the parameters of fig. 3a $\mathcal{D}$ results 0.83 and $\Theta=30$ yields $\mathcal{D}=0.78$

Let us remark that also for very large values of $N_{c}$ some fractal structures may be present, in particular, on very small scales (detectable only for infinitesimally close particles). However, we do not consider this feature because on those scales in real fluids we expect the presence of smoothing due to molecular diffusion.

In order to characterize the system behavior, we have also studied the probability density function $P_{N_{c}}(\tau)$. In figs. 6a-d the probability density functions $P_{N_{c}}(\tau)$ corresponding to $N_{c}=3,10,100,1000$ are shown. For large $N_{c}$ (fig. 6c,d) $P_{N_{c}}(\tau)$ displays an asymptotic shape which can be obtained with simple probabilistic arguments (see below, Sect. IV]), whereas in the opposite case (fig. 6a,b) the distributions exhibit sharp peaks in correspondence of the ballistic time and exponential tails indicating the possibility of very large excursions.

The above results show that the dispersion process in a finite size system cannot be described in terms of a unique characteristic time. As shown in figs. 3a,b, for $N_{c}=3-10$ the exit time $\tau\left(x_{0}, y(0)\right)$ exhibits very strong fluctuations within $3-4$ orders of magnitude, which prevents the possibility to extract meaningful information just from the average of $\tau\left(x_{0}, y(0)\right)$. This is reflected in the exit times probability distribution: for large $N_{c}$ the shape of the distribution suggests the appropriateness of the average exit time to describe 
the diffusion, whereas this is not the case for small $N_{c}$, where $P_{N_{c}}(\tau)$ exhibits exponential tails.

\section{A PROBABILISTIC MODEL COMPARED WITH NUMERICAL RESULTS}

In the previous Section we have discussed some statistical properties of the Lagrangian dynamics generated by the streamfunctions (11,.13); it seems thus natural to look for probabilistic models reproducing the above properties.

The Rossby wave flow (fig. 2a) shows two different regions; the central one characterized by ballistic motion (open streamlines) and particle trapping recirculations (closed streamlines) on both sides of the ballistic regime. The meandering jet flow (fig. 2b), in addition to the recirculation and ballistic ones, presents two far field regions moving retrogradely with respect to the jet core. However, with our parameter choice the far field is practically never visited by the tracer particles [17,34] and thus we shall not consider them.

This intrinsic subdivision of the flow fields suggests to build a discrete symbolic picture of the motion: one can label the trapping and ballistic regions with the symbols $T$ and $B$ respectively and then construct a probabilistic model. Since the studied flows are time periodic we express the time in number periods of the perturbation, namely $2 \pi / \omega$.

The simplest conceivable probabilistic model is a Bernoulli scheme such that at each time the particle can be in the states $B$ with probability $p$ or $T$ with probability $1-p$. However, we do not expect such a model to give a good description of the system, because once the length $L$ of the system (i.e. the equivalent of $N_{c}$ ) is fixed, the probability $P_{L}(\tau)$ that at time $\tau \geq L$ the particle exits from the domain depends just on the parameter $p$, and just one free parameter is obviously not enough to characterize the system behavior.

The natural choice for a model which take into account memory effects is a Markov chain [41]. The process is completely defined by the transition matrix $W_{i j}$, i.e. by the

probability to go in one step to the state $j$ starting from the state $i(i, j=B, T)$ which has the properties:

$$
W_{i j} \geq 0 \quad \text { and } \quad \sum_{j} W_{i j}=1
$$

The probabilities $P_{i}$ to be in state $i$ are given by

$$
P_{i}=\sum_{j} P_{j} W_{j i}
$$

Now we consider the following stochastic process for the evolution of a particle

$$
\Delta x(t)= \begin{cases}1 & \text { with probability } W_{i B} \\ 0 & \text { with probability } W_{i T}\end{cases}
$$

where $i$ represents the state visited at time $t-1$ and $\Delta x(t)$ the increment in the position of the particle at time $t$.

Then we can compute the probability $P_{L}(\tau)$ as follows: 


$$
P_{L}(\tau)=\sum_{k=L-1}^{\tau-1} P_{L-1}(k) F_{B B}(\tau-k)
$$

where $F_{B B}(n)$ is the probability of first arrival from state $B$ to the state $B$ in $n$ steps, which obeys the following recursive formula 41]:

$$
F_{B B}(n)=\left(W^{n}\right)_{B B}-\sum_{k=1}^{n-1} F_{B B}(n-k)\left(W^{k}\right)_{B B}
$$

where $W^{k}$ indicates the $k$-th power of the matrix $W$. Applying recursively the (18) yields

$$
P_{L}(\tau)=\sum_{k_{1}=L-1}^{\tau-1} F_{B B}\left(\tau-k_{1}\right) \sum_{k_{2}=L-2}^{k_{1}-1} F_{B B}\left(k_{1}-k_{2}\right) \ldots \sum_{k_{L_{1}}=1}^{k_{L-2}-1} F_{B B}\left(k_{L-2}-k_{L-1}\right) P_{1}\left(k_{L-1}\right)
$$

being

$$
P_{1}(k)=\left\{\begin{array}{cc}
P_{B} & k=1 \\
P_{T}\left(W_{T T}\right)^{k-2} W_{T B} & k \geq 2
\end{array}\right.
$$

Even if, in general a one-step Markov process is not enough for a detailed description of statistical properties of the dynamical system [17,42,43], it may result a good description as long as $\tau$ is not too small: one indeed expects that for large exit times the memory effects are less important. The expression (20) for $P_{L}(\tau)$ has been compared with the exit time probabilities numerically computed in Sect. [1]. In order to carry out the comparison between the probabilistic model and the numerical results we have first to evaluate the parameters of the model, i.e. $L, P_{i}, W_{i, j}$.

In the numerical evaluation of the matrix $W_{i j}$ and the probabilities $P_{i}$ we have proceeded according to the following scheme. Expressing the time in number of periods of the perturbation, the transition probabilities are computed from a long trajectory $\mathbf{x}_{0}, \mathbf{x}_{1}, \cdots, \mathbf{x}_{n}$ $(n \gg 1)$ in an infinite system $\left(N_{c}=\infty\right)$ as

$$
W_{i j}=\lim _{n \rightarrow \infty} \frac{N_{n}(i, j)}{N_{n}(i)}
$$

where $N_{n}(i)$ is the number of times that, along the trajectory, the particle visits the state $i(i=T$ or $B)$ and $N_{n}(i, j)$ is the number of times that $\mathbf{x}_{t}$ is in state $i$ and $\mathbf{x}_{t+1}$ is in state $j(i, j=T, B)$. The visit probabilities $P_{i}$ are simply given by $\lim _{n \rightarrow \infty} N_{n}(i) / n$. The identification of the visited state is performed by controlling the value of the stream function and the sign of the velocity along the $x, y$-axis [17].

In order to evaluate $L$ we have to take into account that in the physical system the spatial increment per time step (i.e. during one period of the perturbation), $\Delta x$ may vary with time. We have then computed the most probable value $\Delta \tilde{x}$, of $x_{t+(2 \pi / \omega)}-x_{t}$, hence we have rescaled $L$ using $[L / \Delta \tilde{x}]=\tilde{L}$, the square brackets $[\cdot]$ here indicate the integer part of the argument.

In figs. 6a-b the probability density distributions $P_{L}(\tau)$ are compared with those obtained using (20). The small shift of the peak at the beginning of the distributions could depend 
on the estimate of $\Delta \tilde{x}$. As can be seen from the figures 6a-b, even for $L \sim 3-10$ we obtain a good description of $P_{L}(\tau)$ at least for $\tau \gg 1$, i.e. for those particles that experience a large number of transitions between different states. Increasing $L, P_{L}(\tau)$ is better and better approximated by the Markov chain prediction. It is worth remarking that we are comparing the numerical results with the prediction of the probabilistic model without performing any data fitting.

On space/time scales much larger than the typical time/ space scales of the velocity field the evolution of a test particle follows a diffusive scenario. Thus we expect the following process to be a good model for our systems at least in the limit $L \gg 1$ and $\tau \gg 1$ : consider the diffusive stochastic process

$$
x(t)-x(0)=\bar{v} t+\sqrt{2 D} w(t)
$$

where

$$
\left.\bar{v}=\lim _{t \rightarrow \infty}\left\langle\frac{(x(t)-x(0))}{t}\right\rangle \quad \text { and } \quad D=\lim _{t \rightarrow \infty} \frac{1}{2 t}<(x(t)-x(0)-\bar{v} t)^{2}\right\rangle
$$

$w(t)$ is a Wiener process, i.e. a Gaussian process with $w(0)=0$ and

$$
\langle w(t)\rangle=0, \quad\left\langle w(t) w\left(t^{\prime}\right)\right\rangle=\min \left[t, t^{\prime}\right]
$$

Defining $\tau$ the first exit time i.e. the maximum time for which

$$
x(\tau) \leq x_{\max }=\bar{v} \tau+\sqrt{2 D} w(\tau)
$$

the probability density of $\tau$ can be calculated [44] obtaining

$$
P_{x_{\max }}(\tau)=\frac{\left|x_{\max }\right|}{\sqrt{4 \pi D \tau^{3}}} \exp \left[-\frac{\left(\bar{v} \tau-x_{\max }\right)^{2}}{4 D \tau}\right] .
$$

The maximum of $P_{x_{\max }}(\tau)$ is reached for $\tau_{\max }$ which can be estimated, if $D / \bar{v}$ is not too large, as

$$
\tau_{\max } \simeq \frac{x_{\max }}{\bar{v}}
$$

The quantities $\bar{v}$ and $D$ can be evaluated in terms of the exit time statistics as follows

$$
\bar{v}=\frac{L}{<\tau>_{e}} \quad D=\frac{\left\langle(L-\bar{v} \tau)^{2}\right\rangle_{e}}{2<\tau\rangle_{e}}
$$

where the $\langle\cdot\rangle_{e}$ indicates the average over the ensemble of $N_{p}$ particles, i.e. $\langle f\rangle_{e}=$ $\left(1 / N_{p}\right) \sum_{i=1}^{N_{p}} f_{i}$.

In figs. 6a-d the probability distributions calculated in Section III are compared, at different values of $L=x_{\max }$, with the results given by (27) with $\bar{v}$ and $D$ obtained by (29) for large $N_{c}$.

The larger $L$, the better is the fitting with numerical data, nevertheless the probability density function (27) does not capture the tail behavior of the physical system probability $P_{L}(\tau)$. This is not very surprising because the process described by eq. 23. is $\delta$-correlated in time and thus it is not able to describe long-range correlated events [17] responsible of such a tail. Moreover, at variance with the physical system, in model (23) the velocity does not have any bound. 


\section{CONCLUSION AND DISCUSSIONS}

In this paper we have studied non asymptotic properties for passive tracer transport. For a finite system (of size $L$ ), which is a rather common case in real problems (e.g. geophysical and plasma flows), the usual characterization by means of asymptotic quantities (such as the eddy diffusivity) is not appropriate if $L$ is not very large with respect to the typical length scale of the velocity field.

We have considered two models of geophysical interest (traveling waves and meandering jet) studying their Lagrangian transport properties at varying the longitudinal size $L$. Transport has been analyzed in terms of the statistical properties of the exit times of particles from the systems. This analysis has been carried out borrowing concepts from chaotic scattering theory.

In the limit of very large $L$ the usual asymptotic scenario is recovered, i.e. the mean velocity $\bar{v}$ and the diffusion coefficient $D$ completely characterize the transport process. In this case, one typical time is enough to describe the basic features of the process. On the contrary, in the more interesting (and realistic) cases with a not very large $L$, there is no unique characteristic time. Indeed the exit times show a strong sensitivity to initial conditions (this is a manifestation of transient chaos in a non-chaotic system), limiting the possibility of a detailed forecasting of particle behavior. This limitation is mirrored in the probability distribution function of the exit times which displays rather long tails, making impossible the characterization in terms of a unique time (i.e. the average exit time).

Suitable processes (e.g. Markov chains) prove to capture the relevant statistical aspects of transport process. If $L$ is very large the exit times statistics is well described in terms of first exit time problem for a linear Langevin equation involving only $\bar{v}$ and $D$. For systems with a moderate number of recirculation zones one has to introduce a more detailed probabilistic model.

\section{ACKNOWLEDGEMENTS}

We thank M. Falcioni, G. Lacorata and P. Muratore Ginanneschi for useful suggestions and discussions. A particular acknowledgement to B. Marani for the continuous and warm encouragement. We are grateful to the ESF-TAO (Transport Processes in the Atmosphere and the Oceans) Scientific Programme for providing meeting opportunities. This paper has been partly supported by INFM (Progetto di Ricerca Avanzato PRA-TURBO), CNR, MURST (no. 9702265437), and the European Network Intermittency in Turbulent Systems contract number FMRX-CT98-0175. 


\section{REFERENCES}

[1] W.R. Young, Arrested shear dispersion and other models of anomalous diffusion. $J$. Fluid Mech. 193, 129 (1988).

[2] P.B. Rhines and W.R. Young, Homogenization of potential vorticity in planetary gyres. J. Fluid Mech. 122, 347 (1982).

[3] R.S. Pickart, Entrainment and homogenization of passive tracer in a numerical model gyre. J. Geophys. Res. 93, 6761 (1988).

[4] K.J. Richards, Y. Jia and C.F. Rogers, Dispersion of tracers by ocean gyres. J. Phys. Oceanogr. 25, 873 (1995)

[5] G. Buffoni, P. Falco, A. Griffa and E. Zambianchi, Dispersion processes and residence times in a semi-enclosed basin with recirculating gyres: an application to the Tyrrhenian sea. J. Geophys. Res. 102, 18699 (1997).

[6] H. Brenner, Dispersion resulting from flow trough spatially periodic porous media. Philos. Trans. R. Soc. London A297, 81 (1980).

[7] D.L. Koch and J.F. Brady, Dispersion in fixed beds. J. Fluid Mech. 154, 399 (1985).

[8] U. Hornung, Models for flow and transport through porous media derived by homogenization. In M.F. Wheeler (ed.), Environmental studies: Mathematical, computational and statistical analysis, (Spinger, New York, 1996).

[9] D. B. Olson, Rings in the Ocean. Ann. Rev. Earth Planet. Sci. 19, 283 (1991).

[10] A.S. Bower, H.T. Rossby and J.T. Lillibridge, The Gulf Stream - barrier or blender? J. Phys. Oceanogr. 15, 24 (1985).

[11] J.R.N. Lazier and K.H. Mann, Dynamics of Marine Ecosystems. (Blackwell, Cambridge MA, 1994).

[12] G.I. Taylor, Dispersion of soluble matter in solvent flowing slowly trough a tube. Proc. R. Soc. London A219, 186 (1953).

[13] R. Sabot and M.A. Dubois, Diffusion coefficient in a finite domains from exit times and application to the tokamak magnetic structures. Phys. Lett. A212, 201 (1996).

[14] V. Artale, G. Boffetta, A. Celani, M. Cencini and A. Vulpiani, Dispersion of passive tracers in closed basins: beyond the diffusion coefficient. Phys. Fluids. 9, 3162 (1997).

[15] G. Lacorata, E. Aurell and A. Vulpiani, Data analysis and modeling of Lagrangian drifter in the Adriatic sea. J. Marine Res., submitted (1998).

[16] J.H. Misguich, J.D. Reuss, Y. Elskens and R. Balescu, Motion in a stochastic layer described by symbolic dynamics. Chaos 8, 248 (1998).

[17] M. Cencini, G. Lacorata, A. Vulpiani and E. Zambianchi, Mixing in a meandering jet: a Markovian approximation. J. Phys. Oceanogr. in press (1999) chao-dyn/9801027

[18] E.M. Ziemniak, C. Jung and T. Tél, Tracer dynamics in open hydro-dynamical flows as chaotic scattering. Physica D 76, 123 (1994).

[19] G. Károlyi and T. Tél, Chaotic tracer scattering and fractal basin boundaries in a blinking vortex-sink system. Phys. Rep. 290, 125 (1997).

[20] Z. Neufeld and T. Tél, Advection in chaotically time-dependent open flows. Phys. Rev. E 57, 2832 (1997).

[21] A. Crisanti, M. Falcioni, G. Paladin and A. Vulpiani, Lagrangian Chaos: Transport, Mixing and Diffusion in Fluids. La Rivista del Nuovo Cimento 14, 1 (1991).

[22] P. Castiglione, A. Crisanti, A. Mazzino, M. Vergassola and A. Vulpiani, Resonant enhanced diffusion in time-dependent flow. J. Phys. A, 31, 7197 (1998). 
[23] G. Paladin and A. Vulpiani, Anomalous scaling in multifractal object. Phys. Rep. 156, 147 (1987).

[24] E. Aurell, G. Boffetta, A. Crisanti, G. Paladin and A. Vulpiani, Growth of nonifinitesimal perturbations in turbulence. Phys. Rev. Lett. 77, 1262 (1996);

E. Aurell, G. Boffetta, A. Crisanti, G. Paladin and A. Vulpiani, Predictability in the large: an extension of the concept of Lyapunov exponent. J. Phys., A30, 1 (1997).

[25] G.M. Zaslavsky, D. Stevens and H. Weitzner, Self-similar transport in incomplete chaos. Phys. Rev. E, 48, 1683 (1993).

[26] E. Ott, Chaos in dynamical systems. (Cambridge University Press, Cambridge MA, 1993).

[27] R.W. Easton, J.D. Meiss and S. Carver, Exit times and transport for Symplectic Twist Maps. Chaos 3, 153 (1993).

[28] J.H. LaCasce and K.G. Speer, Lagrangian statistics in unforced barotropic flows. J. Marine Res., submitted (1998).

[29] R.T. Pierrehumbert, Chaotic mixing of tracers and vorticity by modulated traveling Rossby Waves. Geophys. Astrophys. Fluid Dynamics 58, 285 (1991).

[30] D. del Castillo Negrete and P.J. Morrison, Chaotic transport by Rossby waves in shear flow. Phys. Fluids A5, 948 (1993).

[31] J. Duan and S. Wiggins, Fluid exchange across a meandering jet with a quasiperiodic variability. J. Phys. Oceanogr., 26, 1176 (1996).

[32] D.R. Watts, Gulf Stream variability. in A.Robinson (ed.), Eddies in Marine Science. (Springer Verlag, Berlin, 1983).

[33] A.S. Bower, A simple kinematic mechanism for mixing fluid parcels across a meandering jet. J. Phys. Oceanogr. 21, 173 (1991).

[34] R.M. Samelson, Fluid exchange across a meandering Jet. J. Phys. Oceanogr. 22, 431 (1992).

[35] S. Dutkiewicz, A. Griffa and D.B. Olson, Particle Diffusion in a Meandering Jet. J. Geophys. Res. 98, 16487 (1993).

[36] B. Cushman-Roisin, Trajectories in Gulf Stream meanders. J. Geophys. Res. 98, 2543 (1993).

[37] G. Lacorata, R. Purini, A. Vulpiani and E. Zambianchi, Dispersion of passive tracers in model flows: effects of the parameterization of small-scale processes. Ann. Geophysicae. 14, 476 (1996).

[38] D.Mc Laughlin, G.C. Papanicolau and O. Pironneau, Convection of microstructure and related problems. SIAM J. Appl. Math.,45, 780, (1985)

[39] A .Bensoussan, J.-L. Lions and G. Papanicolau, Asymptotic Analysis for Periodic Structures (Noth-Holland, Amsterdam, 1978)

[40] P. Grassberger and I. Procaccia, Measuring the strangeness of strange attractors. Physica $D, 9189$ (1983).

[41] W. Feller, An Introduction to Probability theory and its Applications, Vol. I. (Wiley, New York, 1968).

[42] F. Cecconi and A. Vulpiani, Approximation of chaotic systems in terms of Markov processes. Phys. Lett. A201, 326 (1995).

[43] C. Beck and F. Schlögl, Thermodynamics of chaotic systems. (Cambridge University Press, Cambridge, U.K., 1993). 
[44] J.M. Burgers The Nonlinear Diffusion Equation. (D. Reidel Publishing Company, Dordrec, 1974) 


\section{TABLES}

Table 1

Transition matrix elements

\begin{tabular}{|l|l|}
\hline$W_{B B}$ & .66 \\
\hline$W_{B T}$ & .34 \\
\hline$W_{T B}$ & .12 \\
\hline$W_{T T}$ & .88 \\
\hline
\end{tabular}

Table 2

Visit probabilities

\begin{tabular}{|l|l|}
\hline$P_{B}$ & .26 \\
\hline$P_{T}$ & .74 \\
\hline
\end{tabular}

The probabilities are evaluated for the stream function (13) with parameters $k=$ $4 \pi / 15, B_{0}=1.2, c=0.12, \omega=0.4$ and $\gamma=0.3$.

The statistics have been computed over $210^{6}$ periods. 


\section{FIGURE CAPTIONS}

FIGURE 1 :(a) Three realizations of $R^{2}(t)$ built as eq. (9) with: $\gamma=0.08,0.05,0.3$ and $\delta_{0}=10^{-7}, D=1.5$.

(b) $\overline{R^{2}(t)}$ as function of $t$ averaged on the three realizations shown in figure 1a. The apparent anomalous regime and the diffusive one are shown.

(c) $\lambda(\delta)$ vs $\delta$, with Lyapunov and diffusive regimes.

FIGURE 2 :(a) Streamlines for the time-independent Rossby wave flow with: $A_{0}=L_{0}=$ $K_{0}=1, c_{0}=0.5, \alpha=0$. The labels $T$ and $B$ refer to the trapping regions (recirculations) and ballistic ones (the jet channel)

(b) The same as figure (a) for the meandering jet flow with: $k=4 \pi / 15, B_{0}=1.2, c=$ 0.12 and $\gamma=0$.

FIGURE $3 \tau\left(x_{0}, y(0)\right)$ for the meandering jet flow with parameters for the unperturbed streamfunction as fig. $2 \mathrm{~b}$ and $\omega=0.4, \gamma=0.3 \theta=\pi / 2$, and $x_{0}=0.1, y(0) \in[-3,3]$ for (a) $N_{c}=3$ cells, (b) $N_{c}=10$, (c) $N_{c}=100$ and (d) (a) $N_{c}=100$.

FIGURE 4 Two enlargements of figure 3a: (a) for $y(0) \in[1.15: 1.20]$ and (b) $y(0) \in$ [1.1910: 1.1925$]$.

FIGURE 5 Correlation integral $C(r)$ versus $r$ computed from the data of fig. 3a, with threshold $\Theta=15$. The straight line has slope $\mathcal{D}=0.83$.

FIGURE $6 P_{N_{c}}(\tau)$ versus $\tau$ for the meandering jet flow with the same parameters as in fig.3, computed with $410^{4}$ particles starting in $x(0) \in[0, \pi / k], y(0) \in[-3,3]$ for (a) $N_{c}=3$ cells,(b) $N_{c}=10$, (c) $N_{c}=100$ and (d) $N_{c}=1000$. The prediction (27) is shown as dashed continuous line in all the cases. The parameters $\bar{v}$ and $D$ have been calculated from (29) for $N_{c}=1000$ and evaluated as 0.365 and 1.038 respectively. In figure (a) and (b) the Markovian prediction is also shown as continuous lines using $\Delta \tilde{x}=1.36$ and evaluating the probabilities $P_{i}$ and $W_{i j}$ as reported in tables 1 and 2 . 


\section{FIGURES}

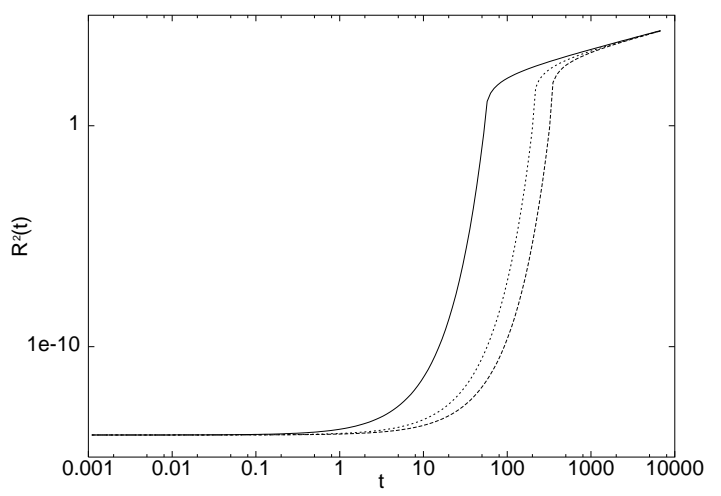

(a)

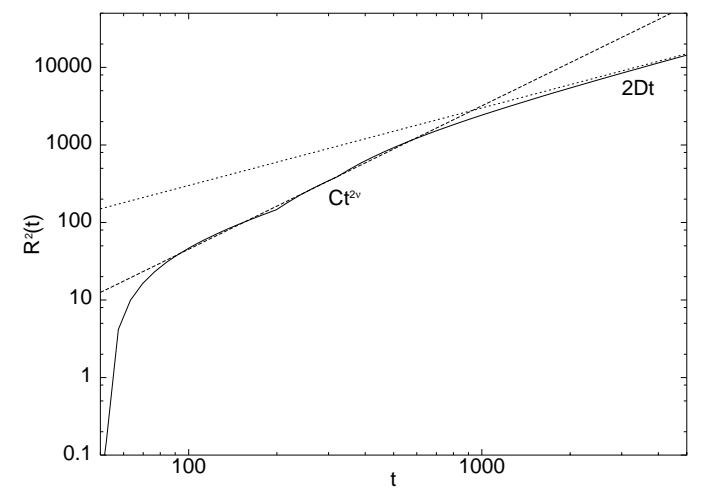

(b)

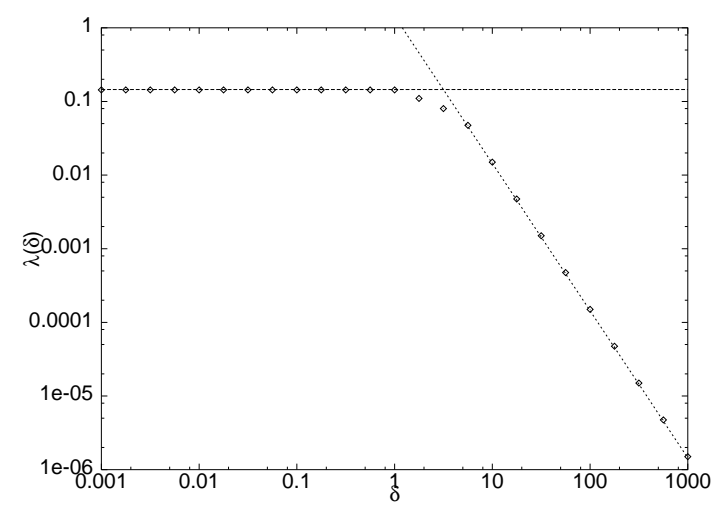

(c)

FIG. 1. 


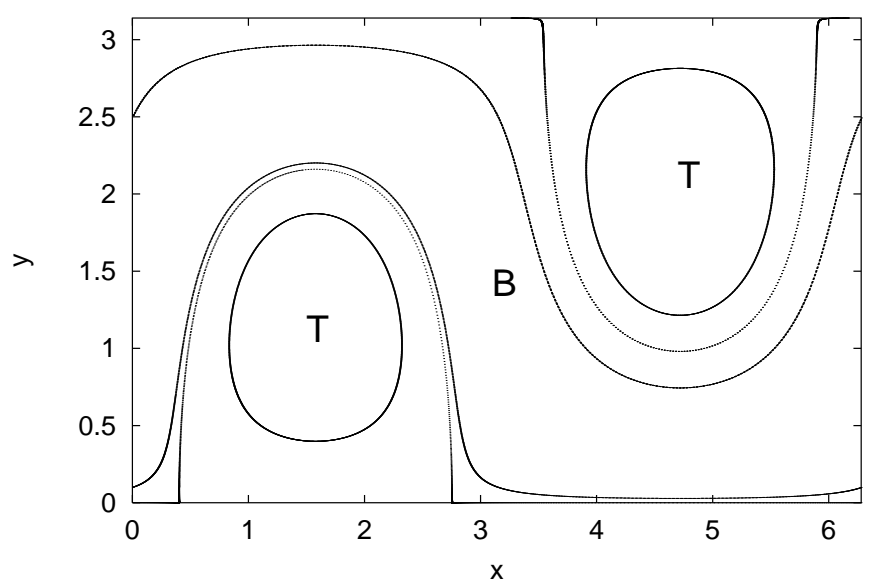

(a)

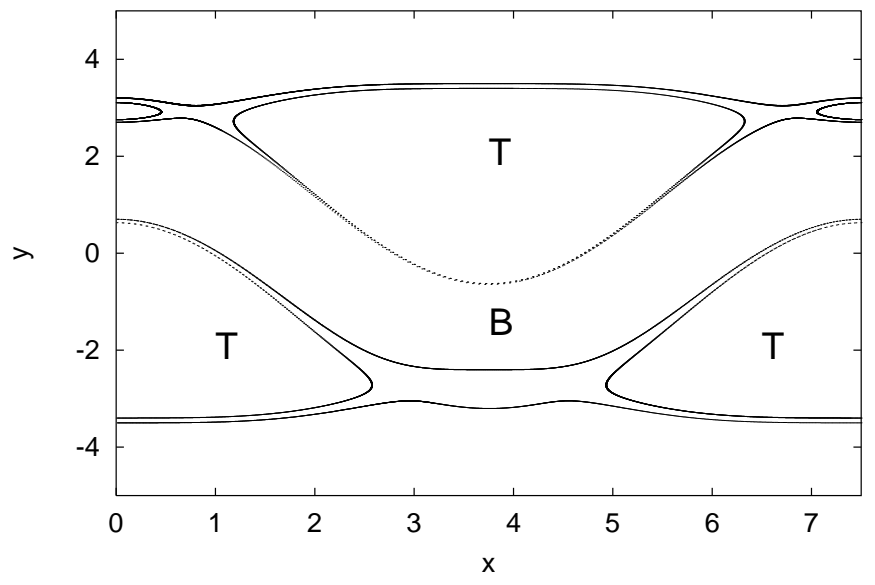

(b)

FIG. 2. 
(a)

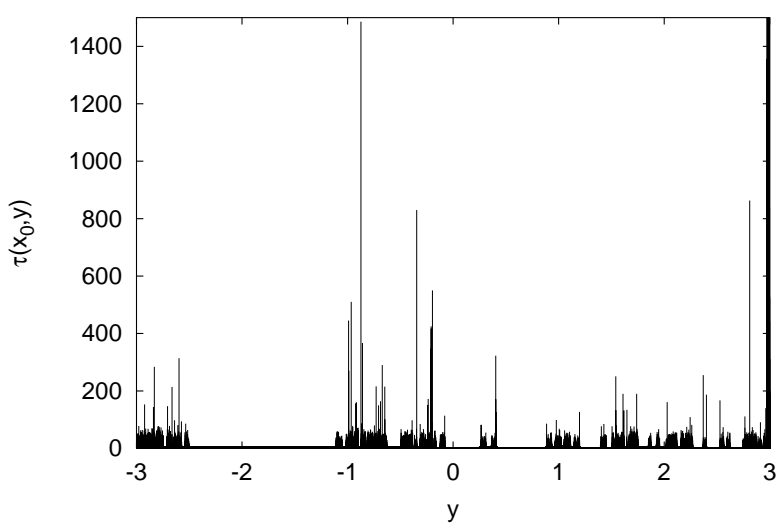

(c)

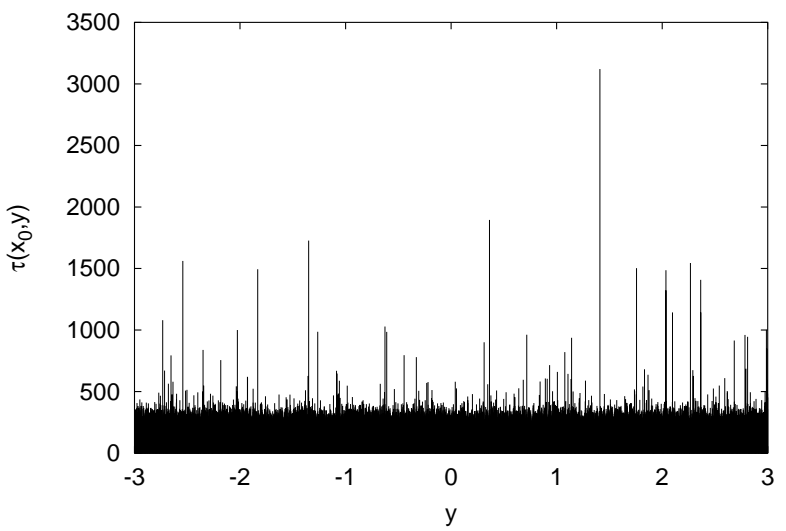

(b)

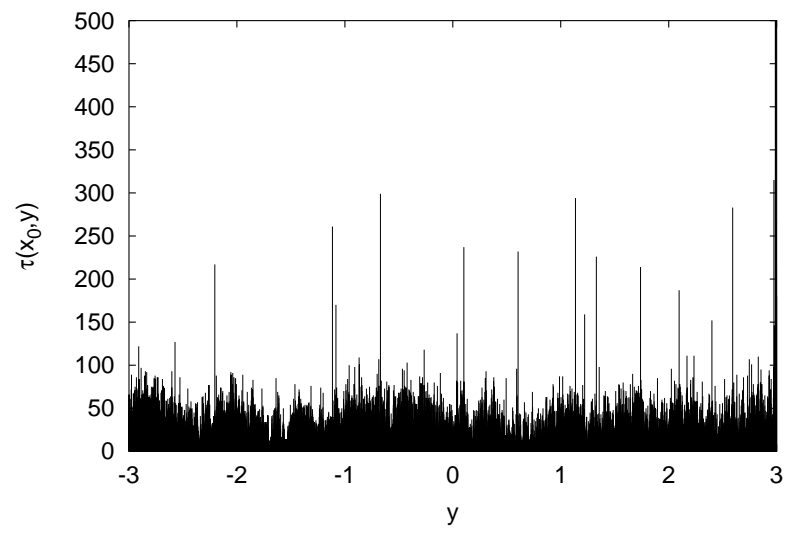

(d)

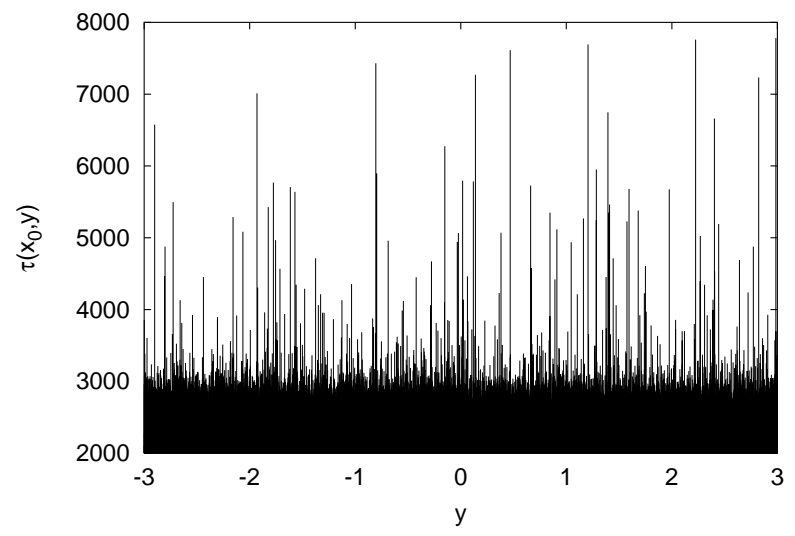

FIG. 3. 


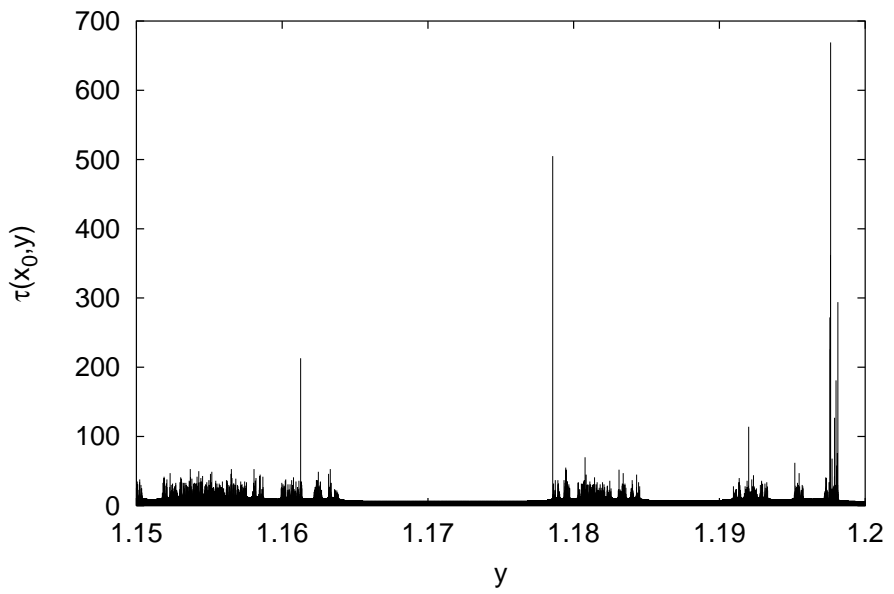

(a)

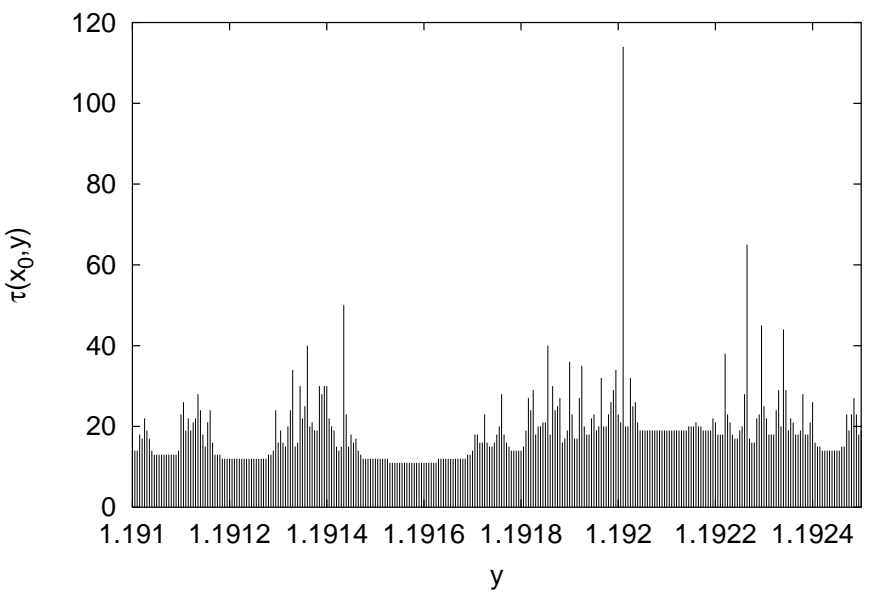

(b)

FIG. 4. 


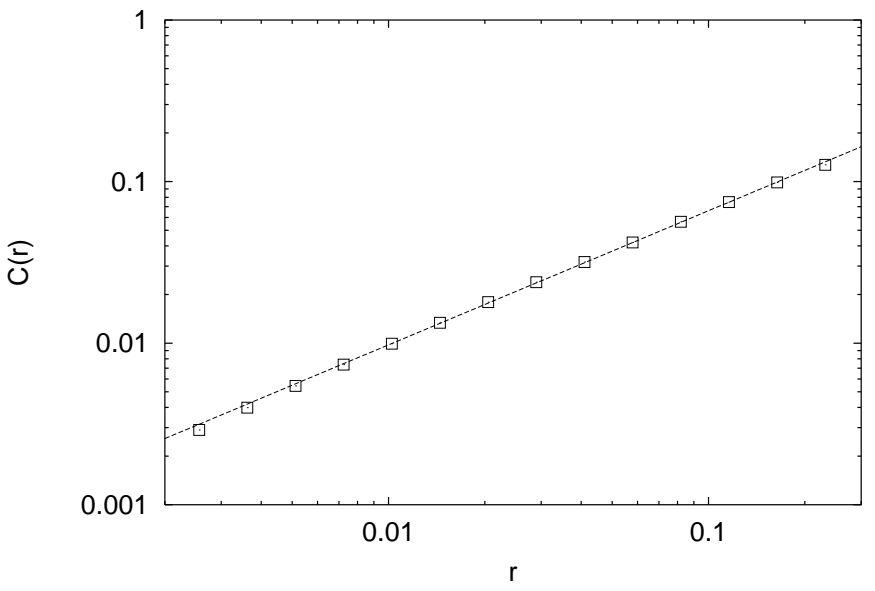

FIG. 5 . 
(a)

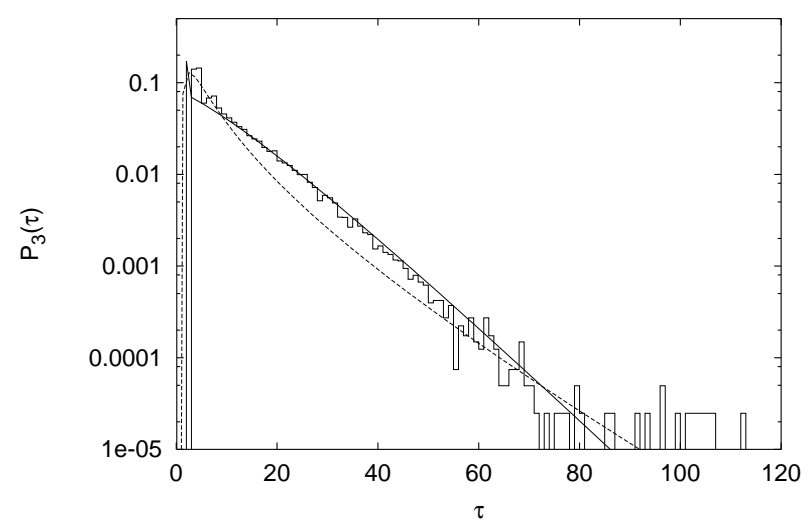

(c)

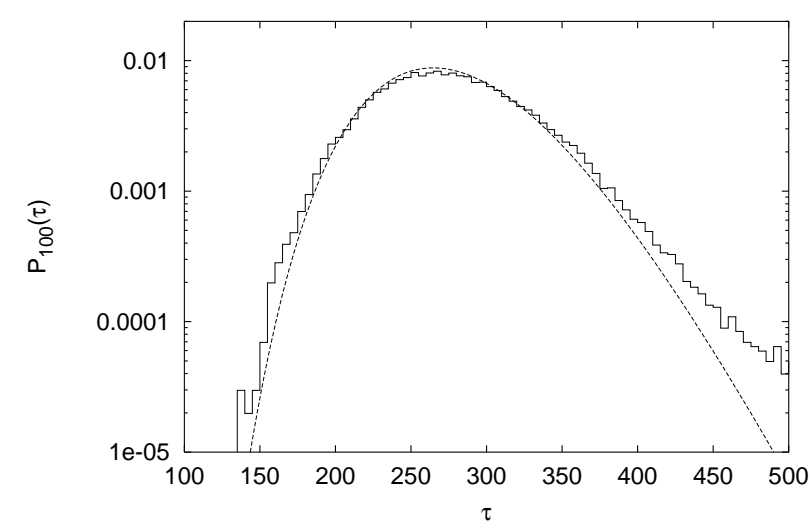

(b)

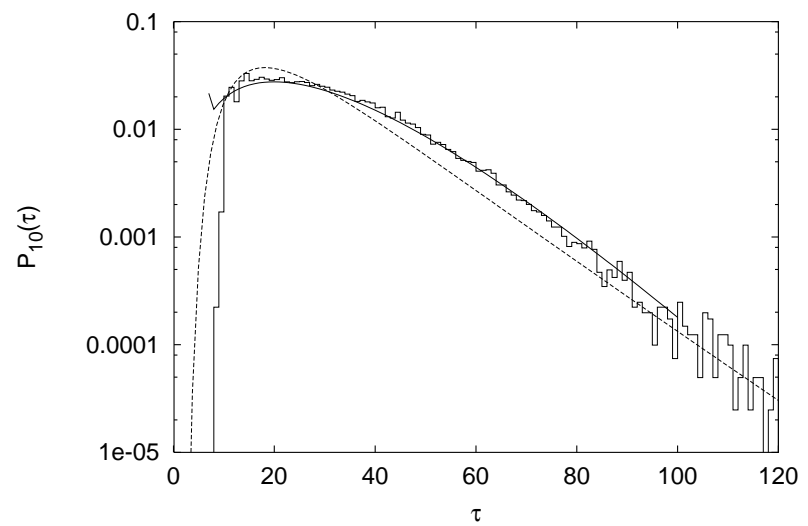

(d)

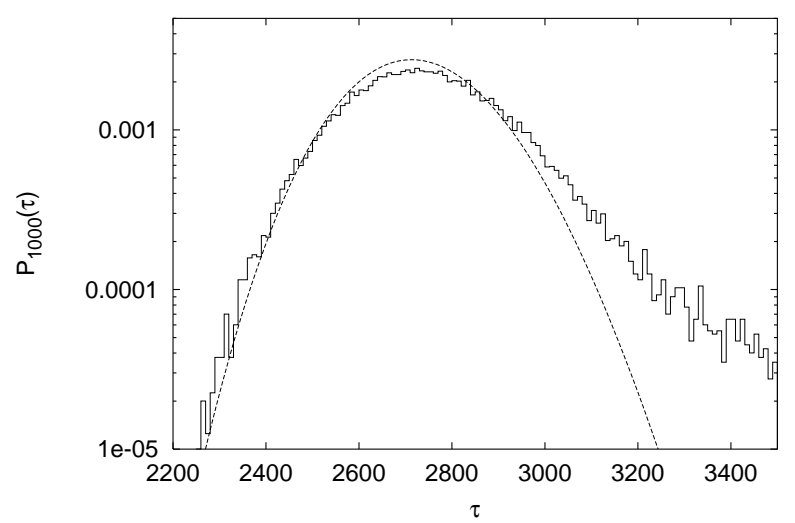

FIG. 6. 